Tohoku J. Exp. Med., 2004, 202, 105-112

\title{
Reliability and Validity of Classification of Senile Postural Deformity in Mass Examinations
}

\author{
Naoyuki Oi, Yoshiko Tobimatsu, ${ }^{1}$ Tsutomu Iwaya, ${ }^{2}$ Yasuhiro Okada, ${ }^{1}$ Satoshi \\ Gushiken, ${ }^{1}$ Shusuke Kusano, Mitsuru Yamamoto, Yasuyuki Takakura and \\ Tetsuo Suyama \\ Department of Rehabilitation, Saitama Medical Center, Saitama Medical \\ School, Kawagoe 350-8550, \\ ${ }^{I}$ Department of Rehabilitation Medicine for People with Physical Disability, \\ Tohoku University Graduate School of Medicine, Sendai 980-8575, and \\ ${ }^{2}$ National Rehabilitation Center for the Disabled, Tokorozawa 359-8555
}

\begin{abstract}
Oi, N., Tobimatsu, Y., Imaya, T., Okada, Y., Gushiken, S., Kusano, S., Yamamoto, M., Takakura, Y. and Suyama, T. Reliability and Validity of Classification of Senile Postural Deformity in Mass Examinations. Tohoku J. Exp. Med., 2004, 202 (2), 105-112 — Nakada (1988) divided senile postural deformities into four types by visual observation: an extended type, an S-shaped type, a flexed type, and a hand-on-the-lap type. The purpose of this study was to investigate the inter-rater reliability and the discriminant validity of assessing the elderly spinal posture using a posture-measuring device developed by us and dividing postural deformities into the four types of Nakada's classification. Seventy-seven elderly persons (52 women and 25 men) who lived independently participated in the study. The average age of the subjects was 73 years (range, 65 to 84 years). The type of the senile postural deformity was determined by three judges using our posture-measuring device in combination with Nakada's classification. The rate of agreement of the classification was $92.2 \%$. This method had a significantly high rate of inter-rater reliability. The thoracic kyphotic angle was larger in the S-shaped type than in the normal, extended type, and flexed type. The lumbar lordotic angle was also larger in the S-shaped type than in the extended type, flexed type, and hand-on-the-lap type. In the hand-onthe-lap type, the mean of the lumbar lordotic angle was much smaller. The lumbosacral angle was smaller in the extended type than in the normal, S-shaped type, and flexed type. With the analysis of x-ray photographs, this method appeared to have discriminant validity as a measure of senile postural deformity. The combination of our posture-measuring device and Nakada's classification would be useful to classify senile postural deformities in mass examinations. —— elderly person; Nakada's classification; senile postural deformity; spinal posture; anteroposterior curves of spine

(C) 2004 Tohoku University Medical Press
\end{abstract}

Received October 8, 2003; revision accepted for publication December 18, 2003.

Address for reprints: Naoyuki Oi, Department of Rehabilitation, Saitama Medical Center, Saitama Medical School, 1981 Tsujido-cho, Kamoda, Kawagoe 350-8550, Japan.

e-mail: spine_oi@saitama-med.ac.jp 
a

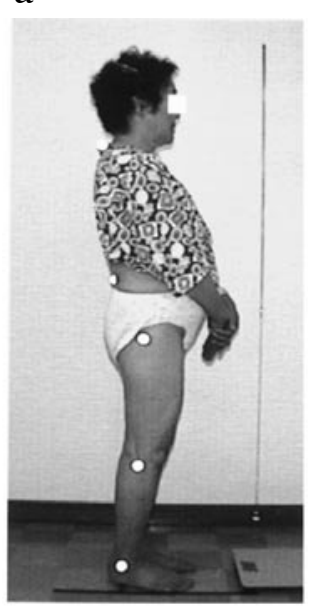

c

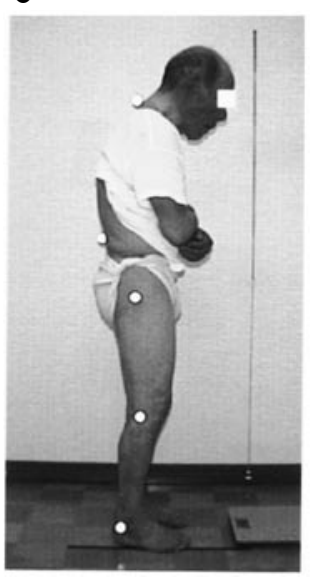

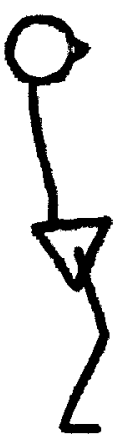

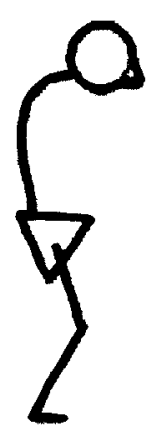

b

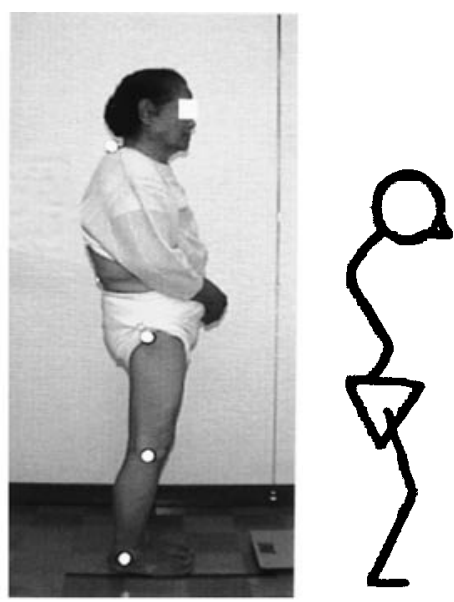

d

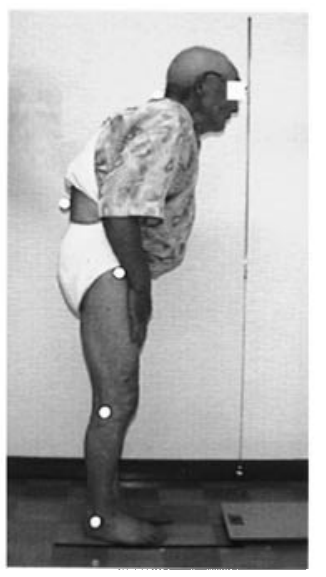

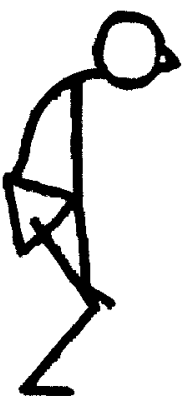

Fig. 1. Nakada's classification of senile postural deformity.

a: extended type, the back was straight and bent backwards.

b: S-shaped type, thoracic kyphosis and lumbar lordosis were severer than in the normal posture.

c: flexed type, the back was rounder than normal and the head was displaced relatively forward.

$\mathrm{d}$ : hand-on-the-lap type, the roundness of the back was severer than in the flexed type and the hands were put on the lap.

Many classifications of senile postural deformities have been proposed during the past 100 years. Staffel's classification (1889) was often quoted in studies of postural deformity in Japan. Wiles (1937) proposed four types of senile posture classified according to the combination of the pelvic inclination and dorsolumbar kyphosis: lumbar lordosis, flat back, sway back, and round back. Round back was divided into two different types according to the lower lumbar curve. However, these classifications were seldom used in a mass examination because of their complex- ity.

Nakada (1988) divided senile postural deformities into four types by visual observation: an extended type, an S-shaped type, a flexed type, and a hand-on-the-lap type (Fig. 1). In the extended type, the back was straight and bent backwards. In the S-shaped type, thoracic kyphosis and lumbar lordosis were severer than in the normal posture. In the flexed type, the back was rounder than normal and the head was displaced relatively forward. In the hand-on-the-lap type, the roundness of the back was severer than in the 
flexed type and the hands were put on the lap.

Classification of the posture in the elderly is important because of the possibility that the ability to participate in various activities may be influenced by the posture. Actually, we studied the relation between the functional status of the elderly and the posture using Nakada's classification. The score of the instrumental activity of daily life criterion was lower in the hand-on-thelap group than in the other groups (Oi et al. 1998) and maximum walking speed in the hand-on-thelap group was lower than that in the other groups (Okada et al. 2000). Although Nakada's classification is simple and useful for field studies, its reliability and validity have not been proven.

The purpose of this study was to investigate the inter-rater reliability and the discriminant validity of assessing the elderly spinal posture using a posture-measuring device developed by us and dividing postural deformities into the four types of Nakada's classification.

\section{Materials ANd Methods}

The study included elderly persons each of whom lived independently in rural areas of the northern part of Japan and received a general medical examination. Seventy-seven persons (52 women and 25 men) participated in the study. The average age of the persons was 73 years (range, 65 to 84 years).

The type of senile postural deformity was determined using our posture-measuring device in combination with Nakada's classification. The posture-measuring device was made of aligned aluminium tubes put into two boards so that wooden rods ( $88 \mathrm{~cm}$ length and $3 \mathrm{~mm}$ in diameter) could move freely through the tubes (Fig. 2). The participants stood in their most comfortable posture with their backs to the side of the posturemeasuring device. The joint angles of lower extremities were allowed to remains as they were. The examiner put the tips of the wooden rods to the spinous processes of the participants. Then, the contour of the other ends of the rods was photographed and three judges who had knowledge

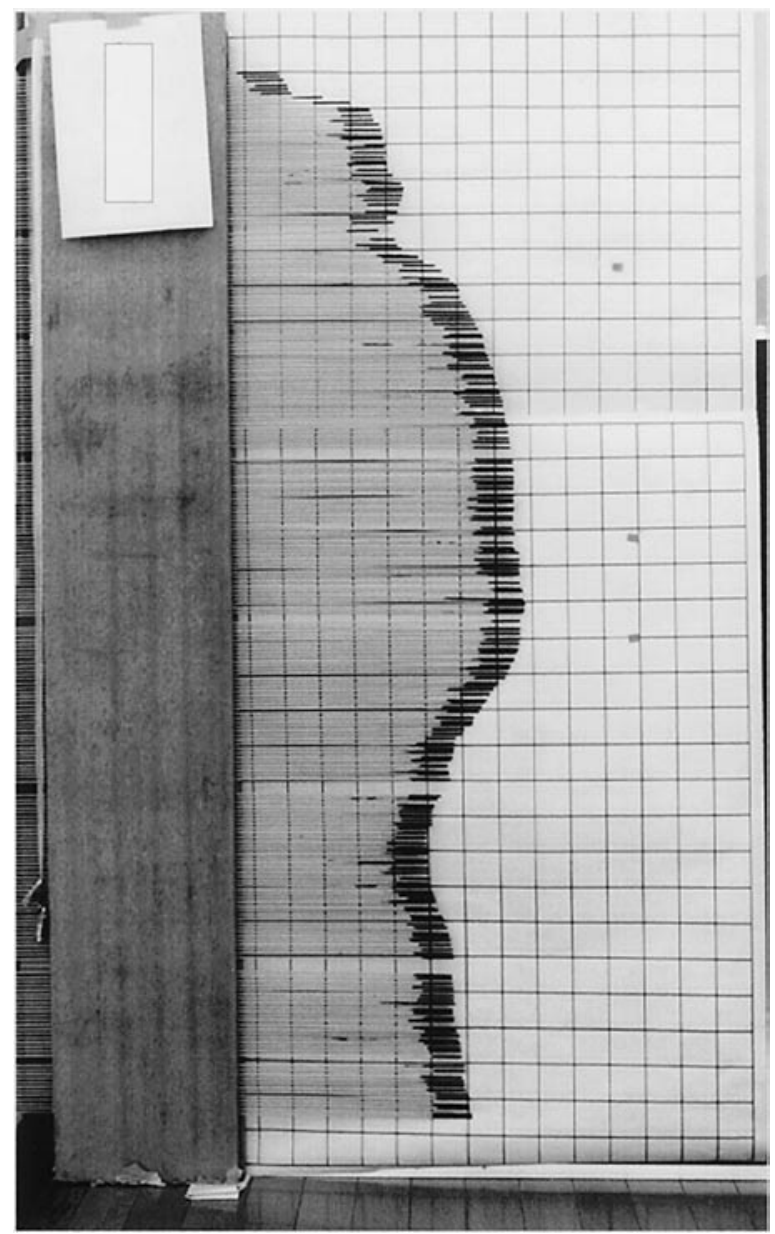

Fig. 2. Assessing the elderly spinal posture using our posture-measuring device.

The posture-measuring device was made of aligned aluminium tubes put into two boards so that wooden rods could move freely through the tubes. The participants stood with their backs to the side of the posture-measuring device. The examiner put the tips of the rods to the spinous processes of the participants. A photograph of the contour of the other ends of the rods shows the posture of the participant. This participant was classified into the flexed type group by unanimous agreement of all three judges.

of Nakada's classification determined the type of senile postural deformity from these pictures.

$x$-Ray photographs of the lateral view of the whole spine were taken. The participants were asked to stand with their usual posture with their right sides facing the $\mathrm{x}$-ray films. The thoracic kyphotic angle, lumbar lordotic angle, and lumbo- 


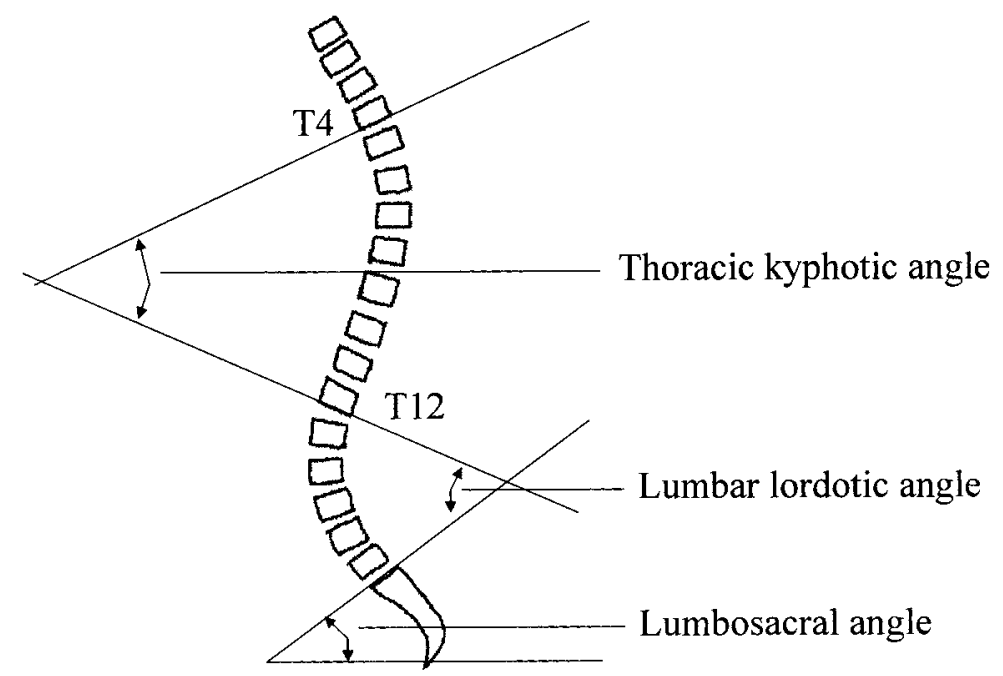

Fig. 3. Measurements of spinal deformity on the lateral x-ray photographic images.

The thoracic kyphotic angle was the angle obtaining between the inferior edge of the T4 vertebral body and the T12 vertebral body. The lumbar lordotic angle was the angle between the inferior edge of the T12 vertebral body and the superior edge of the S1 vertebral body. The lumbosacral angle was the angle between the superior edge of the S1 vertebral body and a horizontal line.

sacral angle (Hellems and Keats 1971) were measured on the lateral X-ray photographic images of the thoracic, lumbar, and sacral spine (Fig. 3). The thoracic kyphotic angle was the angle obtaining between the inferior edge of the T4 vertebral body and the T12 vertebral body. The lumbar lordotic angle was the angle between the inferior edge of the T12 vertebral body and the superior edge of the S1 vertebral body. The lumbosacral angle was the angle between the superior edge of the $\mathrm{S} 1$ vertebral body and a horizontal line.

The type of senile postural deformity was determined based on the pictures taken while using the posture-measuring device. A participant's posture was classified into a postural type when at least two of the three judges agreed on the determination. The rate of agreement of the classification was calculated. After excluding participants who could not be ascribed a certain posture type, the significance of the differences in the thoracic kyphotic angle, lumbar lordotic angle, and lumbosacral angle among each group of senile postural deformities was statistically analyzed using oneway analysis of variance and post-hoc tests. SPSS 9.0J (SPSS Japan Inc., Tokyo) was used for the statistic analyses. The statistically significant level was set at less than 5\%.

Although we did not obtain institutional ethics committee approval for the study, all of the procedures were conducted in accordance with the Helsinki Declarations of 1975. We obtained informed consent to publish the photographs from the participants.

\section{RESUlTS}

Seventy-one participants were able to be classified into a certain type of senile postural deformity by the agreement of at least two of the three judges. The rate of agreement was $92.2 \%$.

TABLE 1. The number of the participants of each postural group

\begin{tabular}{lcc}
\hline & $\begin{array}{c}\text { Number of } \\
\text { participants }\end{array}$ & $\begin{array}{c}\text { Average age } \\
\text { (years) }\end{array}$ \\
\hline Normal & 26 & $69.0 \pm 4.4$ \\
Extended type & 8 & $75.5 \pm 4.1$ \\
S-shaped type & 14 & $73.6 \pm 4.4$ \\
Flexed type & 21 & $74.9 \pm 5.0$ \\
Hand-on-the-lap type & 2 & $82.0 \pm 1.4$ \\
\hline
\end{tabular}




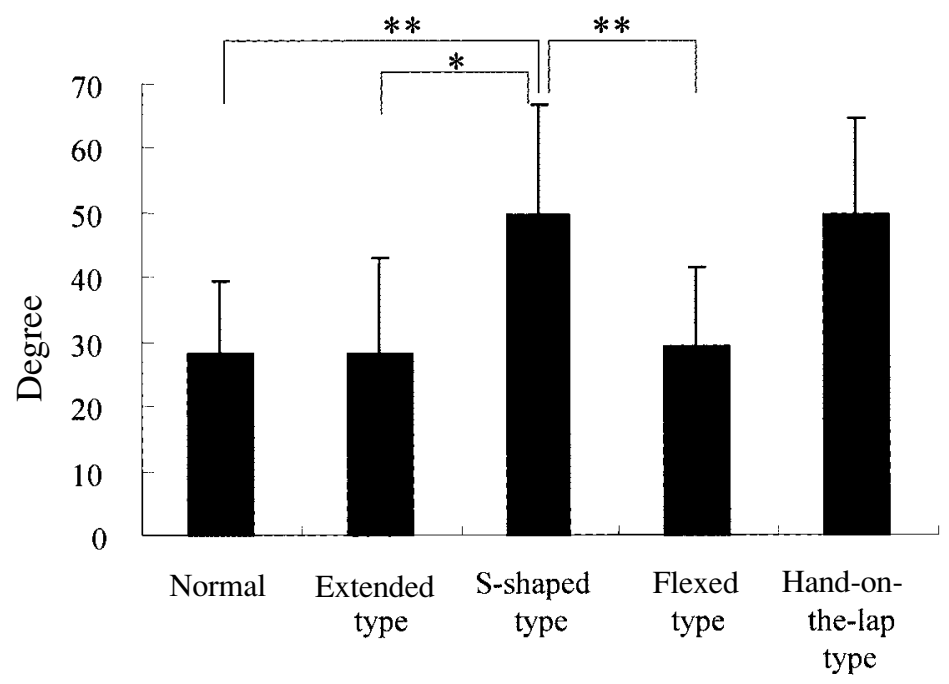

Fig. 4. Average value of the thoracic kyphotic angle in each postural type. ${ }^{*} p<0.05^{* *}, p<0.01$.

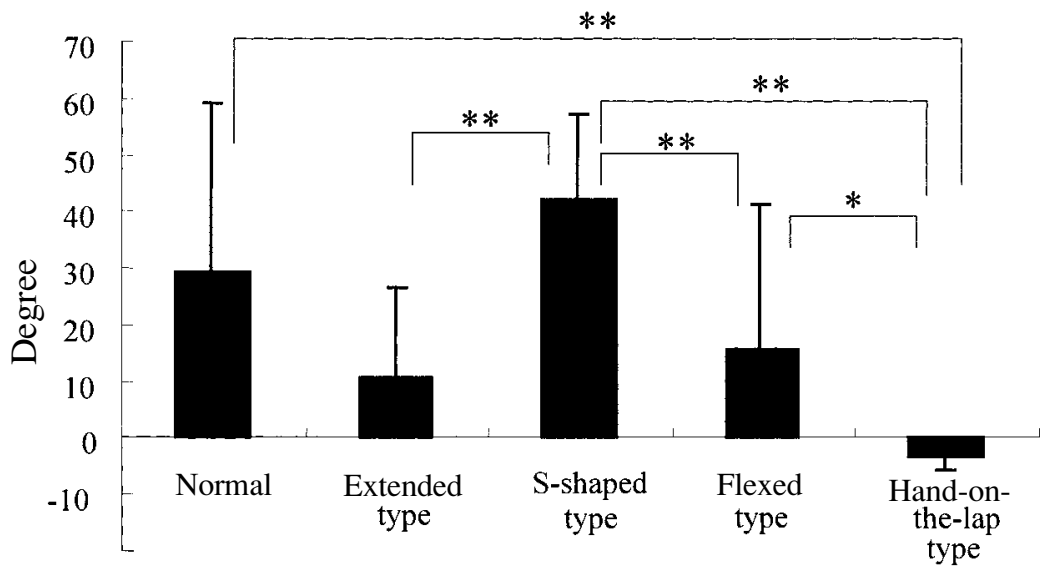

Fig. 5. Average value of the lumbar lordotic angle in each postural type. * $p<0.05^{* *}, p<0.01$.

Thirty-seven of the participants were classified into a certain group by unanimous agreement of all three judges. The number of the participants of each group is shown in Table 1. In the analysis of $\mathrm{x}$-ray photographs, the thoracic kyphotic angle was significantly larger in the S-shaped type than in the normal, extended type, and flexed type. $\left(\mathrm{F}_{[4,64]}=7.557 p<0.01\right.$, Scheffe's post-hoc test [Fig. 4]). The lumbar lordotic angle was also significantly larger in the S-shaped type than in the extended type, flexed type, and hand-on-thelap type. In the hand-on-the-lap type, the mean of the lumbar lordotic angle was much smaller, and the lumbar lordosis disappeared $\left(\mathrm{F}_{[4,65]}=3.962\right.$ $p<0.01$, Tamhane's post-hoc test. [Fig. 5]). The lumbosacral angle was smaller in the extended type than in the normal, S-shaped type, and flexed type $\left(\mathrm{F}_{[4,65]}=2.793 p<0.05\right.$, Tamhane's post-hoc test [Fig. 6]).

\section{Discussion}

The standing posture of humans varies dynamically with the surroundings. Although there is a wide range of normal thoracic kyphosis and 


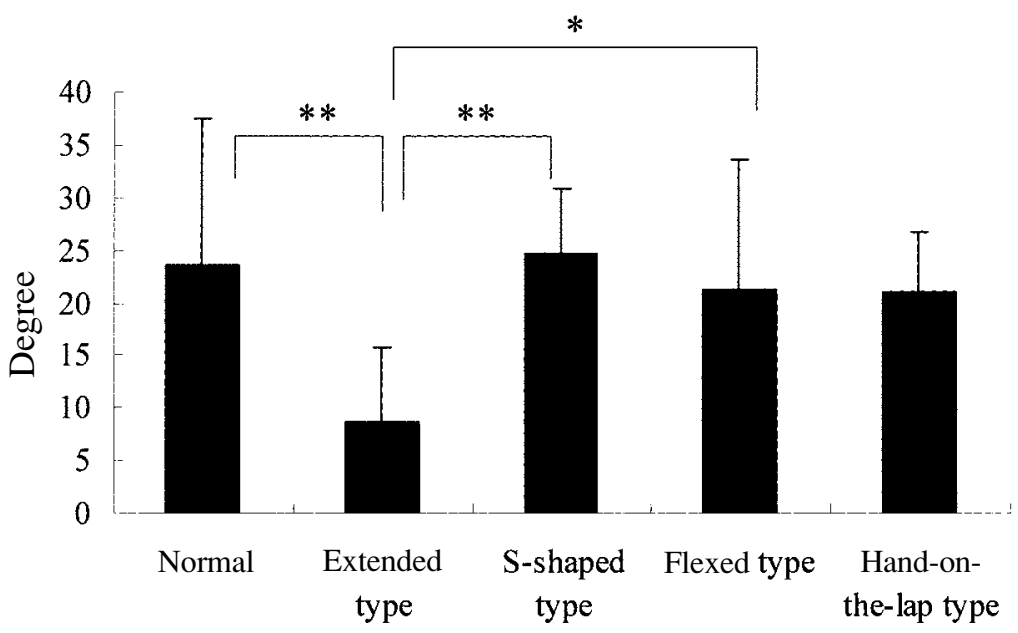

Fig. 6. Average value of the lumbosacral angle in each postural type. $p<0.05^{* *}, p<0.01$

TABLE 2. The correlation between the data obtained from the X-ray photographs and the type of senile postural deformity

\begin{tabular}{lccccc}
\hline & Normal & Extended type & S-shaped type & Flexed type & $\begin{array}{l}\text { Hand-on-the-lap } \\
\text { type }\end{array}$ \\
\hline Thoracic kyphotic angle & Small & Small & Large & Small & \\
Lumbar lordotic angle & Medium & Small & Large & Small & Very small \\
Lumbosacral angle & Large & Small & Large & Large & \\
\hline
\end{tabular}

lumbar lordosis, the normal sagittal plane curves of the spine tend to balance each other such that the head, trunk, and pelvis are lined up vertically by segmental mobility (Bernhardt and Bridwell 1988; Jackson and McManus 1994; Gelb et al. 1995; Van Royen et al. 1998). The spinal column degenerates with aging due to the progression of osteoporosis which can cause compression fractures of the vertebral body, decreases in the intervertebral disc height, and facet joint stiffness that can restrict dynamic postural changes. A round back posture induces backaches and restricts one's activities in daily life. If the roundness becomes more severe, the ability to walk is decreased. Accordingly, the assessment of senile postural deformities is very important for studying restrictions in daily life of the elderly. However, accurate determinations of posture have been difficult because the classification methods are very complex and the postures of subjects frequently change. Among the methods for classifying posture, Nakada's classification is very simple and it can classify senile postural deformities without any tools or devices. On the other hand, this method has a disadvantage in terms of the reliability and validity. In this study, we studied the inter-rater reliability and the discriminate validity when using our posture-measuring device together with Nakada's classification system.

Ninety-two percent of the participants were classified into a certain type of posture with the agreement of at least two of the three judges. More than $50 \%$ of the participants were able to be classified by unanimous agreement of three judges. From this result, we concluded that this method had a substantially high rate of inter-rater reliability.

The correlation between the data obtained 
from the x-ray photographs and the type of senile postural deformity is summarized in Table 2. It should be noted that only in the S-shaped type were both the thoracic kyphotic and lumbar lordotic angles large. The $\mathrm{S}$-shaped type is a special type of senile posture. It seems that osteoporotic compression fractures of the mid thoracic spine account for the S-shaped posture. A decrease in both the thoracic kyphotic angle and lumbar lordotic angle was observed in the extended type as well as in the flexed type. However, the lumbosacral angle of the extended type was significantly smaller than that of the flexed type. This suggested that the spinal deformities of the extended type and the flexed type were basically the same and that the flexed posture might be compensated with a posterior inclination of the pelvis causing extension of the back. The lumbar lordotic angle was smaller in the hand-on-thelap type than in the flexed type. In this study, the mean of the lumbar lordotic angle in the hand-onthe-lap type was not more than zero degrees. As shown in Table 2, the posture of an elderly person was clearly distinguishable by this method. The discriminate validity of this method using our posture-measuring device together with Nakada's classification system was confirmed.

The posture-measuring device developed by us was useful for observing the standing position of the elderly in a mass examination. Examination methods for mass check-ups should be simple and inexpensive. When observing the posture of the elderly, although it would be better if the patients were undressed, this might not be realistic in a mass examination. Nevertheless, the contour obtained by our device accurately reflected the spinal curvature of the person examined because the tips of the rods were placed on the surface of the clothes contacting the spine directly. In this study, we proved that Nakada's classification reflected the senile postural deformity by comparisons made between X-ray photographs and the spinal contours obtained from our posture-measuring device.

\section{Conclusion}

Nakada's classification is very simple to use for visual classification of senile postural deformities. The combination of our posturemeasuring device and Nakada's classification had a significantly high rate of inter-rater reliability. This method seemed to have discriminant validity as a measure of senile postural deformity. The combination of our posture-measuring device and Nakada's classification will be useful to classify senile postural deformities in mass examinations.

\section{References}

Bernhardt, M. \& Bridwell, K.H. (1988) Segmental analysis of the sagittal plane alignment of the normal thoracic and lumbar spines and thoracolumbar junction. Spine, 14, 717-721.

Gelb, D.E., Lenke, L.G., Bridwell, K.H., Blanke, K. \& McEnery, K.W. (1995) An analysis of sagittal spinal alignment in 100 asymptomatic middle and older aged volunteers. Spine, 20, 1351-1358.

Hellems, H.K. \& Keats, T.E. (1971) Measurement of the normal lumbosacral angle. Am. J. Roentgenol. Radium Ther. Nucl. Med., 113, 642-645.

Jackson, R.P. \& McManus, A.C. (1994) Radiographic analysis of sagittal plane alignment and balance in standing volunteers and patients with low back pain matched for age, sex, and size. Spine, 19, 1611-1618.

Nakada, K. (1988) A study on senile postural deformity. J. Jpn. Orthop. Assoc., 62, 1149-1161 (in Japanese with English abstract).

Oi, N., Iwaya, T., Seki, K., Yoshida, K., Imada, G. \& Urushiyama, Y. (1998) A study on effects of senile postural deformity on functional status. Jpn. J. Rehabil. Med., 35, 1060. (in Japanese)

Okada, Y., Nagaya, N., Otaka, K., Oi, N., Tobimatsu, Y. \& Iwaya, T. (2000) The relation between standing posture and walking ability of healthy elderly people. J. Phys. Med., 11, 118-124 (in Japanese with English abstract).

Staffel, F. (1889) Die menschlichen Haltungstypen und ihre Beziehungen zu den Ruckgratverkrummungen. J. F. Bergman, Wiesbaden. (in Germany)

Van Royen, B.J., Toussaint, H.M., Kingma, I., Bot, S.D.M., Caspers, M., Harlaar, J. \& Wuisman, P.I.J.M. (1998) Accuracy of the sagittal vertical 
axis in a standing lateral radiograph as a measurement of balance in spinal deformities. Eur. Spine J., 7, 408-412.
Wiles, P. (1937) Postural deformities of the anteroposterior curves of the spine. Lancet, 17, 911-919. 